\title{
Quasiconformal homogeneity of hyperbolic manifolds
}

\author{
Petra Bonfert-Taylor • Richard D. Canary • Gaven Martin • Edward Taylor
}

Received: 5 May 2004 / Published online: 13 November 2004 - (C) Springer-Verlag 2004

\begin{abstract}
We exhibit strong constraints on the geometry and topology of a uniformly quasiconformally homogeneous hyperbolic manifold. In particular, if $n \geq 3$, a hyperbolic $n$-manifold is uniformly quasiconformally homogeneous if and only if it is a regular cover of a closed hyperbolic orbifold. Moreover, if $n \geq 3$, we show that there is a constant $K_{n}>1$ such that if $M$ is a hyperbolic $n$-manifold, other than $\mathbb{H}^{n}$, which is $K$-quasiconformally homogeneous, then $K \geq K_{n}$.
\end{abstract}

Mathematics Subject Classification (2000): $30 \mathrm{C60}$

\section{Introduction}

An (orientable) hyperbolic manifold $M$ is $K$-quasiconformally homogeneous if, given any two points $x, y \in M$, there exists a $K$-quasiconformal homeomorphism $f: M \rightarrow M$ such that $f(x)=y$. If $M$ is $K$-quasiconformally homogeneous for some $K$, we say that it is uniformly quasiconformally homogeneous. In this paper we discuss the geometric and topological constraints on uniformly quasiconformally homogeneous hyperbolic manifolds. In dimensions $n \geq 3$ we will characterize such manifolds. The situation in dimension two is more mysterious.

It is easy to check, see Proposition 2.4 below, that any closed hyperbolic manifold is uniformly quasiconformally homogeneous. Our first result shows that there are rather severe restrictions on the geometry of uniformly quasiconformally homogeneous hyperbolic manifolds. We define $\ell(M)$ to be the infimum of the

P. BONFERT-TAYLOR ${ }^{\star}$, E. TAYLOR ${ }^{\star \star}$

Wesleyan University, Middletown, CT, USA (e-mail: pbonfert@wesleyan.edu; ectaylor@weslesyan. edu)

R. D. CANARY ${ }^{\star \star \star}$

University of Michigan, Ann Arbor, MI 48109, USA (e-mail: canary@umich.edu)

G. MARTIN ${ }^{\star \star \star \star}$

The University of Auckland, Auckland, New Zealand

(e-mail: martin@math.auckland.ac.nz)

* Research supported in part by NSF grant 070335 and 0305704.

$\star \star$ Research supported in part by NSF grant 0203698.

$\star \star \star$ Research supported in part by the NZ Marsden Fund and the Royal Society (NZ).

$\star \star \star \star$ Research supported in part by NSF grant 0305704. 
lengths of homotopically nontrivial curves in $M$ and $d(M)$ to be the supremum of the diameters of embedded hyperbolic balls in $M$.

Theorem 1.1. For each dimension $n \geq 2$ and each $K \geq 1$, there is a positive constant $m(n, K)$ with the following property. Let $M=\mathbb{H}^{n} / \Gamma$ be a $K$-quasiconformally homogeneous hyperbolic n-manifold, which is not $\mathbb{H}^{n}$. Then

(1) $d(M) \leq K \ell(M)+2 K \log 4$.

(2) $\ell(M) \geq m(n, K)$, i.e. there is a lower bound on the injectivity radius of $M$ that only depends on $n$ and $K$.

(3) Every nontrivial element of $\Gamma$ is hyperbolic and the limit set $\Lambda(\Gamma)$ of $\Gamma$ is $\partial \mathbb{H}^{n}$.

As a corollary we see that a geometrically finite hyperbolic $n$-manifold is uniformly quasiconformally homogeneous if and only if it is closed.

Corollary 1.2. A geometrically finite hyperbolic manifold $M$ is uniformly quasi conformally homogeneous if and only if $M$ is closed.

However, there are many noncompact uniformly quasiconformally homogeneous $n$-manifolds. For example, consider the cover $N$ associated to the fiber of a closed hyperbolic 3-manifold $M$ which fibers over the circle. We will see, in Lemma 2.6, that there exists $K$ such that given any two points $x_{1}$ and $x_{2}$ in $M$ there exists a $K$-quasiconformal automorphism of $M$ which is homotopic to the identity and takes $x_{1}$ to $x_{2}$. Let $y_{1}, y_{2} \in N$ and let $z_{i}$ be the projection of $y_{i}$ to $M$. Let $f: M \rightarrow M$ be a $K$-quasiconformal automorphism homotopic to the identity such that $f\left(z_{1}\right)=z_{2}$. Let $\tilde{f}: N \rightarrow N$ be a lift of $f$ to $N$. Then $\tilde{f}\left(y_{1}\right)=y_{2}^{\prime}$ where $y_{2}^{\prime}$ is a pre-image of $z_{2}$. Since $N$ is a regular cover there exists an isometry $h: N \rightarrow N$ such that $h\left(y_{2}^{\prime}\right)=y_{2}$. Then $h \circ \tilde{f}$ is a $K$-quasiconformal map carrying $y_{1}$ to $y_{2}$. Thus, $N$ is $K$-quasiconformally homogeneous.

The argument in the paragraph above generalizes to show that any regular cover of a closed hyperbolic manifold is uniformly quasiconformally homogeneous. One may further show, using a different argument, that any regular cover of a closed hyperbolic orbifold is uniformly quasiconformally homogeneous, see Proposition 2.7. In dimensions 3 and above, we will use McMullen's version of Sullivan's rigidity theorem to show that all uniformly quasiconformally homogeneous hyperbolic manifolds are regular covers of closed hyperbolic orbifolds.

Theorem 1.3. Suppose that $n \geq 3$. A hyperbolic $n$-manifold is uniformly quasiconformally homogeneous if and only if it is a regular cover of a closed hyperbolic orbifold.

We will later see that there exist uniformly quasiconformally homogeneous hyperbolic surfaces that are not regular covers of closed orbifolds (see Lemma 5.1). 
In dimension 3, we will observe that any uniformly quasiconformally homogeneous hyperbolic 3-manifold with finitely generated fundamental group is the cover associated to the fiber of a closed 3-manifold which fibers over the circle; see Theorem 7.1.

Finally, we will show that for hyperbolic manifolds of dimension at least 3, the quasiconformal homogeneity constant is uniformly bounded away from 1. It is unknown whether this result holds in dimension 2.

Theorem 1.4. If $n \geq 3$, there is a constant $K_{n}>1$ such that if $M$ is a $K$-quasiconformally homogeneous hyperbolic n-manifold which is not $\mathbb{H}^{n}$, then $K \geq K_{n}$.

History: Gehring and Palka [6] introduced the related concept of quasiconformal homogeneity for domains in space. A set $\Sigma$ is said to be quasiconformally homogeneous if and only if the group of quasiconformal automorphisms of $\Sigma$ acts transitively on $\Sigma$. MacManus, Näkki and Palka, see $[9,10]$, have studied quasiconformally homogeneous compacta in the plane.

Acknowledgements. We would like to thank Yair Minsky for helpful conversations on the subject matter of this paper.

\section{Basic facts}

We start by defining the quasiconformal homogeneity constant $K(M)$ of a hyperbolic manifold. We observe the convention that $K(M)=\infty$ if $M$ is not uniformly quasiconformally homogeneous.

Lemma 2.1. Let $M$ be a uniformly quasiconformally homogeneous hyperbolic manifold. Then

$$
K(M)=\min \{K \mid M \text { is } K \text {-quasiconformally homogeneous }\}
$$

exists.

Proof of Lemma 2.1. Suppose that $M$ is $K_{j}$-quasiconformally homogeneous for a sequence $\left\{K_{j}\right\}$ converging to $K$. We show that $M$ is $K$-quasiconformally homogeneous which will complete the proof.

Recall that the family $\mathcal{F}_{L}$ of $L$-quasiconformal automorphisms of $\mathbb{H}^{n}$ is a normal family for any $L$ (see Theorem 19.2 and 20.5 of [19].) Let $x, y \in M$. Since $M$ is $K_{j}$-quasiconformally homogeneous for each $j$, there exists a $K_{j}$-quasiconformal mapping $f_{j}: M \rightarrow M$ with $f_{j}(x)=y$. Let $\tilde{x}$ and $\tilde{y}$ be lifts of $x$ and $y$ to $\mathbb{H}^{n}$. Each $f_{j}$ has a lift $\tilde{f}_{j}: \mathbb{H}^{n} \rightarrow \mathbb{H}^{n}$, necessarily $K_{j}$-quasiconformal, such that $\tilde{f}_{j}(\tilde{x})=\tilde{y}$. Then the family $\left\{\tilde{f}_{j}\right\}$ is a normal family, and one can choose a subsequence of $\left\{\tilde{f}_{j}\right\}$ that converges to a $K$-quasiconformal mapping $\tilde{f}: \mathbb{H}^{n} \rightarrow \mathbb{H}^{n}$ which descends to a $K$-quasiconformal mapping $f: M \rightarrow M$ taking $x$ to $y$. 
Hyperbolic space $\mathbb{H}^{n}$ is 1-quasiconformally homogeneous, since its isometry group Isom $+\left(\mathbb{H}^{n}\right)$ acts transitively. In fact, $\mathbb{H}^{n}$ is the only 1-quasiconformally homogeneous hyperbolic manifold:

Proposition 2.2. Let $M$ be a complete hyperbolic n-manifold, so that $\pi_{1}(M) \neq$ \{id\}. Then $M$ is not 1-quasiconformally homogeneous.

The proof of Proposition 2.2 makes use of the following standard fact (see, for example, Proposition V.E.10 in [13]).

Lemma 2.3. If $M=\mathbb{H}^{n} / \Gamma$ is a hyperbolic manifold and $\Gamma$ is nonelementary (i.e. not virtually abelian), then its isometry group $\operatorname{Isom}(M)$ acts properly discontinuously on M.

Proof of Proposition 2.2. Recall that a 1-quasiconformal automorphism of a hyperbolic manifold is an isometry. If $\Gamma$ is nonelementary, then Lemma $2.3 \mathrm{im}-$ plies that the 1-quasiconformal automorphisms of $M$ do not act transitively on $M$.

If $\Gamma$ is elementary it is easy to verify, via the classification of elementary groups, that $\mathbb{H}^{n} / \Gamma$ contains two points $x$ and $y$ with distinct injectivity radii. It follows that the group of isometries cannot act transitively.

Next we show that every closed hyperbolic manifold $M$ is uniformly quasiconformally homogeneous.

Proposition 2.4. Every closed hyperbolic manifold is uniformly quasiconformally homogeneous. Moreover, if $M$ is a closed hyperbolic n-manifold, then

$$
K(M) \leq\left(e^{\frac{\ell(M)}{4}}+1\right)^{2(n-1)\left(\frac{4 \mathrm{diam}(M)}{\ell(M)}+1\right)}
$$

where $\operatorname{diam}(M)$ denotes the diameter of $M$.

Our main tool in the proof of this proposition is a local version of this result which is essentially due to Gehring and Palka:

Lemma 2.5. Suppose that $x, y \in \mathbb{H}^{n}$ and $d(x, y)<\varepsilon$. Let $B$ be the ball of radius $2 \varepsilon$ about $x$. Then there exists a K-quasiconformal mapping $f: \mathbb{H}^{n} \rightarrow \mathbb{H}^{n}$ such that $f(x)=y, f$ agrees with the identity map on an open neighborhood of $\mathbb{H}^{n} \backslash B$, and

$$
K \leq\left(e^{\varepsilon}+1\right)^{2(n-1)} .
$$

Proof of Lemma 2.5. We will work in the ball model $\mathbb{B}^{n}$ and normalize so that $x=0$. Choose $\delta$ such that $d(0, y)<\delta<\epsilon$ and let $B^{\prime}$ be the ball or radius $2 \delta$ about 0 . Lemma 3.2 in Gehring-Palka [6] implies that there exists a $K$-quasiconformal mapping $f: \overline{\mathbb{R}^{n}} \rightarrow \overline{\mathbb{R}^{n}}$ so that $f_{\overline{\mathbb{R}^{n}} \backslash B^{\prime}}=\mathrm{id}, f(0)=y$ and

$$
\log K \leq 2(n-1) k_{B^{\prime}}(0, y),
$$


where $k_{B^{\prime}}$ is the quasihyperbolic metric on $B^{\prime}$. Note that, because $f_{\overline{\mathbb{R}^{n}} \backslash B^{\prime}}=\mathrm{id}$ and $B^{\prime} \subset \mathbb{B}^{n}$ we have that $f$ preserves $\mathbb{B}^{n}$.

We now estimate $k_{B^{\prime}}(0, y)$. As the hyperbolic distance between 0 and $y$ is less than or equal to $\delta$, the Euclidean distance between 0 and $y$ is less than or equal to $\frac{e^{\delta}-1}{e^{\delta}+1}$. Furthermore, the Euclidean radius of $B^{\prime}$ is $\frac{e^{2 \delta}-1}{e^{2 \delta}+1}$. Thus

$$
\begin{aligned}
k_{B}(0, y) & \leq \int_{0}^{\frac{e^{\delta}-1}{e^{\delta}+1}} \frac{1}{\frac{e^{2 \delta}-1}{e^{2 \delta}+1}-t} d t \\
& =\log \left(e^{\delta}+1\right)+\log \left(\frac{e^{2 \delta}-1}{2\left(e^{2 \delta}-e^{\delta}\right)}\right) \\
& \leq \log \left(e^{\delta}+1\right) \\
& \leq \log \left(e^{\epsilon}+1\right) .
\end{aligned}
$$

Therefore, $K \leq\left(e^{\varepsilon}+1\right)^{2(n-1)}$ as claimed.

Proof of Proposition 2.4. Let $M$ be a hyperbolic $n$-manifold and let $\varepsilon=\ell(M) / 4$ and $L=\left(e^{\varepsilon}+1\right)^{2(n-1)}$.

We first verify the proposition for points which are less than $\varepsilon$ apart. Let $x$ and $y$ be two points in $M$ such that $d(x, y)<\varepsilon$. Choose a covering map $\pi: \mathbb{H}^{n} \rightarrow M$ and let $B$ be the ball of radius $2 \varepsilon$ about $x$. By the definition of $\varepsilon$, there exists a local section $s: B \rightarrow \mathbb{H}^{n}$. Let $\tilde{x}=s(x)$ and $\tilde{y}=s(y)$. Lemma 2.5 implies that there exists a $L$-quasiconformal map $g: \mathbb{H}^{n} \rightarrow \mathbb{H}^{n}$ such that $g(\tilde{x})=\tilde{y}$ and $g$ agrees with the identity map on an open neighborhood of $\mathbb{H}^{n} \backslash B$. We may then define a $L$-quasiconformal map $f: M \rightarrow M$ such that $f(x)=y$ by setting $f=\pi \circ g \circ s$ on $B$ and $f=$ id elsewhere.

If $x, y \in M$ are two arbitrary points then there exists a chain of points $\left\{z_{0}, z_{1}, \ldots, z_{m}\right\}$ in $M$ such that $d\left(z_{i-1}, z_{i}\right)<\varepsilon$ for all $i, z_{0}=x, z_{m}=y$, and $m<\frac{\operatorname{diam}(M)}{\varepsilon}+1$. For each $i$, we construct a $L$-quasiconformal map $f_{i}: M \rightarrow M$ such that $f_{i}\left(z_{i-1}\right)=z_{i}$. If we set $h=f_{m} \circ \cdots \circ f_{1}$, then $h(x)=y$ and $h$ is $L^{m}$-quasiconformal. It follows that

$$
K(M) \leq L^{\frac{\operatorname{diam}(M)}{\varepsilon}+1} .
$$

Notice that each $f_{i}$, and hence $h$, is homotopic to the identity.

The argument in the last paragraph of the proof of Proposition 2.4 gives the following lemma:

Lemma 2.6. Let $M$ be a hyperbolic n-manifold and let $x, y \in M$. If there exists a path $\alpha$ in $M$ of length $R$ joining $x$ to $y$ such that every point on $\alpha$ has injectivity radius at least $2 \varepsilon$, then there exists a $K$-quasiconformal map $f: M \rightarrow M$ which is homotopic to the identity such that $f(x)=y$ and

$$
K \leq\left(e^{\varepsilon}+1\right)^{2(n-1)\left(\frac{R}{\varepsilon}+1\right)} .
$$


We now show that regular covers of closed hyperbolic orbifolds are uniformly quasiconformally homogeneous. We recall that an orientable closed hyperbolic $n$-orbifold is a compact quotient $Q=\mathbb{H}^{n} / \Gamma$ where $\Gamma$ is a discrete group of orientation-preserving isometries of $\mathbb{H}^{n}$. A hyperbolic manifold $M$ is a regular cover of $Q$, if $M=\mathbb{H}^{n} / \Gamma_{0}$ where $\Gamma_{0}$ is a normal, torsion-free subgroup of $\Gamma$.

Proposition 2.7. Any regular cover of a closed hyperbolic orbifold is uniformly quasiconformally homogeneous. Moreover, if $M$ is a regular cover of a closed hyperbolic n-orbifold $Q=\mathbb{H}^{n} / \Gamma$, then

$$
K(M) \leq\left(e^{\frac{\ell^{\prime}(Q)}{4}}+1\right)^{2(n-1)\left(\frac{4 \operatorname{diam}(Q)}{\ell^{\prime}(Q)}+1\right)}
$$

where $\ell^{\prime}(Q)$ is the minimal translation length of a hyperbolic element of $\Gamma$ and $\operatorname{diam}(Q)$ is the diameter of $Q$.

Notice that the bound on $K(M)$ depends only on the geometry of $Q$.

Proof of Proposition 2.7. Since $M=\mathbb{H}^{n} / \Gamma_{0}$ is a regular cover of $Q$, there exists a group $G$ of isometries of $M$ such that $Q=M / G$. Recall that every element of $G$ is a conformal map of $M$.

Since $Q$ is closed, $\ell^{\prime}(Q)>0$ and we let $\varepsilon=\ell^{\prime}(Q) / 4$. Every nontrivial element of $\Gamma_{0}$ is hyperbolic and has translation length at least $4 \varepsilon$. It follows that every point in $M$ has injectivity radius at least $2 \varepsilon$.

Let $y_{1}, y_{2} \in M$. As $M$ is a regular cover of $Q$ there exists $g \in G$ such that $d\left(g\left(y_{1}\right), y_{2}\right) \leq \operatorname{diam}(Q)$. Lemma 2.6 then implies that there exists a $K$-quasiconformal map $f: M \rightarrow M$ such that $f\left(g\left(y_{1}\right)\right)=y_{2}$ and

$$
K=\left(e^{\varepsilon}+1\right)^{2(n-1)(\operatorname{diam}(Q) / \varepsilon+1)} .
$$

Then $h=f \circ g$ is a $K$-quasiconformal homeomorphism such that $h\left(y_{1}\right)=y_{2}$. It follows that $M$ is $K$-quasiconformally homogeneous.

\section{Geometric constraints}

The purpose of this section is to develop the geometric consequences of uniform quasiconformal homogeneity. We first recall a well-known result which is a consequence of the Margulis lemma, see [22,12].

Lemma 3.1. For each $n \geq 2$ there exists $d_{n}>0$ such that if $M$ is a hyperbolic n-manifold, then

$$
d(M) \geq d_{n},
$$

i.e. every hyperbolic n-manifold contains an embedded hyperbolic ball of diameter at least $d_{n}$. 
Remark 3.2. In the classical case $n=2$ the precise value of $d_{n}$ was found by Yamada [23], while in dimension 3 an explicit estimate is given in [5].

We next recall that quasiconformal maps are $(K, K \log 4)$-quasi-isometries (in the hyperbolic metric), see, for example Theorem 11.2 in [20]. However, quasiconformal maps need not be bilipschitz.

Lemma 3.3. Let $f: \mathbb{H}^{n} \rightarrow \mathbb{H}^{n}$ be a $K$-quasiconformal homeomorphism. Then

$$
\frac{1}{K} d(x, y)-\log 4 \leq d(f(x), f(y)) \leq K d(x, y)+K \log 4
$$

for all $x, y \in \mathbb{H}^{n}$.

Since quasiconformal maps of hyperbolic manifolds lift to quasiconformal maps of $\mathbb{H}^{n}$, the same result holds for any hyperbolic manifold.

Lemma 3.4. Let $M$ be a hyperbolic n-manifold and let $f: M \rightarrow M$ be a $K-$ quasiconformal homeomorphism. Then

$$
\frac{1}{K} d(x, y)-\log 4 \leq d(f(x), f(y)) \leq K d(x, y)+K \log 4
$$

for all $x, y \in M$.

The additive constants $\log 4$ and $K \log 4$ in Lemma 3.3 make this inequality less useful for nearby points. The estimate below provides uniform estimates on the distortion of points which are close together, see Theorem 11.2 in [20].

Lemma 3.5. Let $f: \mathbb{H}^{n} \rightarrow \mathbb{H}^{n}$ be a $K$-quasiconformal homeomorphism. Then

$$
\tanh \left(\frac{d(f(x), f(y))}{2}\right) \leq \lambda_{n}^{(1-J)}\left(\tanh \left(\frac{d(x, y)}{2}\right)\right)^{J}
$$

for all $x, y \in \mathbb{H}^{n}$ where $J=K^{1 /(1-n)}$ and $\lambda_{n} \in\left[4,2 e^{n-1}\right)$ is the Grötzsch constant.

We are now prepared for the proof of Theorem 1.1 which we restate for the reader's convenience.

Theorem 1.1. For each dimension $n \geq 2$ and each $K \geq 1$, there is a positive constant $m(n, K)$ with the following property. Let $M=\mathbb{H}^{n} / \Gamma$ be a $K$-quasiconformally homogeneous hyperbolic n-manifold, which is not $\mathbb{H}^{n}$. Then

(1) $d(M) \leq K \ell(M)+2 K \log 4$.

(2) $\ell(M) \geq m(n, K)$, i.e. there is a lower bound on the injectivity radius of $M$ that only depends on $n$ and $K$.

(3) Every nontrivial element of $\Gamma$ is hyperbolic and the limit set $\Lambda(\Gamma)$ of $\Gamma$ is $\partial \mathbb{H}^{n}$. 
Proof of Theorem 1.1. Let $x$ lie on a homotopically nontrivial closed curve $\alpha$ in $M$ of length $l$ and let $y$ be the center of an embedded hyperbolic ball of radius $r$ in $M$. Since $M$ is $K$-quasiconformally homogeneous, there exists a $K$-quasiconformal map $f: M \rightarrow M$ such that $f(x)=y$. As $f(\alpha)$ is homotopically nontrivial, there exists $f(z) \in f(\alpha)$ such that $d(y, f(z)) \geq r$. Lemma 3.4 implies that

$$
r \leq d(f(x), f(z)) \leq K d(x, z)+K \log 4 \leq K l / 2+K \log 4 .
$$

Since $l$ may be chosen to be arbitrarily close to $\ell(M)$ and $r$ may be chosen arbitrarily close to $d(M) / 2$ inequality (1) follows.

If we use Lemma 3.5 instead of Lemma 3.4, we see that

$$
\tanh \left(\frac{d(M)}{2}\right) \leq \lambda_{n}^{(1-J)}\left(\tanh \left(\frac{\ell(M)}{2}\right)\right)^{J}
$$

where $J=K^{1 /(1-n)}$. Since $d(M) \geq d_{n}$, this implies that

$$
\ell(M) \geq m(n, K)=2 \tanh ^{-1}\left(\lambda_{n}^{J-1}\left(\tanh \left(d_{n} / 2\right)\right)^{1 / J}\right)
$$

which establishes (2).

If $\Gamma$ contains a parabolic element, then $\ell(M)=0$ which contradicts (2), so every nontrivial element of $\Gamma$ is hyperbolic. If the limit set of $\Gamma$ is not all of $\partial \mathbb{H}^{n}$, then $M$ contains an embedded hyperbolic half-space. So $d(M)=\infty$ which contradicts (1). This establishes (3).

Since noncompact geometrically finite hyperbolic $n$-manifolds either contains cusps (where the injectivity radius gets arbitrarily close to 0 ) or hyperbolic halfspaces (whether the injectivity radius gets arbitrarily large), we obtain the following corollary:

Corollary 1.2. A geometrically finite hyperbolic manifold $M$ is uniformly quasiconformally homogeneous if and only if $M$ is closed.

Proof of Corollary 1.2. Proposition 2.4 gives that closed hyperbolic manifolds are uniformly quasiconformally homogeneous. Suppose that $M=\mathbb{H}^{n} / \Gamma$ is uniformly quasiconformally homogeneous. Theorem 1.1, part (2), implies that $\Gamma$ contains no parabolic elements. If $\Gamma$ is geometrically finite and has no parabolic elements, then its limit set consists entirely of conical limit points (see BeardonMaskit [2] or Apanasov [1]). Since a Dirichlet fundamental polyhedron cannot accumulate at a conical limit point (Proposition B.5 in [13]) and $\Lambda(\Gamma)=\partial \mathbb{H}^{n}$, the Dirichlet fundamental polyhedron of $\Gamma$ must be compact, so $M$ must be closed. 


\section{Distortion estimates for quasiconformal maps}

We recall (see, for example, Theorem 17.18 in [19]) that every quasiconformal homeomorphism of $\mathbb{H}^{n}$ extends continuously to a homeomorphism of $\mathbb{H}^{n} \cup \partial \mathbb{H}^{n}$. We first see that if the extension of a quasiconformal map is the identity on $\partial \mathbb{H}^{n}$ then it is uniformly close to the identity map on $\mathbb{H}^{n}$.

Lemma 4.1. For all $n \geq 2$, there is an increasing function $\psi_{n}:(1, \infty) \rightarrow(0, \infty)$ with the following property. If $f: \mathbb{H}^{n} \rightarrow \mathbb{H}^{n}$ is $K$-quasiconformal, the extension of $f$ to $\partial \mathbb{H}^{n}$ is the identity map, and $x \in \mathbb{H}^{n}$, then $d(x, f(x)) \leq \psi_{n}(K)$. Moreover, $\lim _{K \rightarrow 1^{+}} \psi_{n}(K)=0$.

Proof of Lemma 4.1. Though this result is known (e.g. see [4]), we sketch a proof for the reader's convenience. Fix $n \geq 2$. Working in the unit ball model $\mathbb{B}^{n}$ we recall that the family $\mathcal{F}_{K}$ of all $K$-quasiconformal mappings $f: \mathbb{B}^{n} \rightarrow \mathbb{B}^{n}$ with $f \mid \partial \mathbb{B}^{n}=$ id is compact, and set

$$
\psi_{n}(K)=\max \left\{d(0, f(0)) \mid: f \in \mathcal{F}_{K}\right\} .
$$

For each $K>1$, let $f_{K}: \mathbb{B}^{n} \rightarrow \mathbb{B}^{n}$ be a $K$-quasiconformal map such that $d\left(f_{K}(0), 0\right)=\psi_{n}(K)$ and $\left.f_{K}\right|_{\partial \mathbb{B}^{n}}=\mathrm{id}$. Notice that if $f_{1}$ is the limit of a sequence $\left\{f_{K_{j}}\right\}$ where $K_{j}$ converges to 1 , then $f_{1}$ is 1 -quasiconformal and $\left.f_{1}\right|_{\partial \mathbb{B}^{n}}=$ id, so $f_{1}$ is equal to the identity. It follows that $\lim _{K \rightarrow 1^{+}} \psi_{n}(K)=0$.

Notice that if $x \in \mathbb{H}^{n}, f: \mathbb{H}^{n} \rightarrow \mathbb{H}^{n}$ is $K$-quasiconformal and the extension of $f$ to $\partial \mathbb{H}^{n}$ is the identity map, then we may identify $\mathbb{H}^{n}$ with $\mathbb{B}^{n}$ so that $x$ is identified with 0 . Therefore, $d(x, f(x)) \leq \psi_{n}(K)$.

We now apply Lemma 4.1, Theorem 1.1 and McMullen's version of Sullivan's rigidity theorem to show that any quasiconformal map of a uniformly quasiconformally homogeneous manifold is uniformly near an isometry.

Proposition 4.2. Suppose that $M$ is a uniformly quasiconformally homogeneous hyperbolic $n$-manifold (with $n \geq 3$ ). If $f: M \rightarrow M$ is a $K$-quasiconformal homeomorphism and $x \in M$, then there exists an orientation-preserving isometry $g$ of $M$ such that

$$
d(g(x), f(x)) \leq \psi_{n}(K) .
$$

Proof of Proposition 4.2. Let $M=\mathbb{H}^{n} / \Gamma$ be a uniformly quasiconformally homogeneous hyperbolic $n$-manifold with $n \geq 3$. Theorem 1.1 implies that $d(M)<\infty$ and $\Lambda(\Gamma)=\partial \mathbb{H}^{n}$. Let $x \in M$ and let $f: M \rightarrow M$ be a $K$-quasiconformal homeomorphism. Theorem 2.10 in McMullen [14] implies that $f$ is homotopic to an orientation-preserving isometry $g: M \rightarrow M$.

Let $H$ be the homotopy between $f$ and $g$. Then $H$ lifts to a homotopy between lifts $\tilde{f}: \mathbb{H}^{n} \rightarrow \mathbb{H}^{n}$ and $\tilde{g}: \mathbb{H}^{n} \rightarrow \mathbb{H}^{n}$ of $f$ and $g$. Given any hyperbolic element $\gamma \in \Gamma$, the lifted homotopy moves any points on (the image of) the axis of $\gamma$ a 
uniformly bounded amount, so the extensions of $\tilde{f}$ and $\tilde{g}$ to $\partial \mathbb{H}^{n}$ agree on the fixed points of $\gamma$. Since fixed points of hyperbolic elements are dense in $\Lambda(\Gamma)=\partial \mathbb{H}^{n}$, the extensions of $\tilde{f}$ and $\tilde{g}$ to $\partial \mathbb{H}^{n}$ agree on $\partial \mathbb{H}^{n}$. It follows that $\tilde{g}^{-1} \circ \tilde{f}$ is $K$-quasiconformal and the extension of $\tilde{g}^{-1} \circ \tilde{f}$ to $\partial \mathbb{H}^{n}$ agrees with the identity map. Lemma 4.1 implies that

$$
d\left(\tilde{g}^{-1}(\tilde{f}(y)), y\right) \leq \psi_{n}(K)
$$

for all $y \in \mathbb{H}^{n}$, so

$$
d\left(g^{-1}(f(x)), x\right)=d(f(x), g(x)) \leq \psi_{n}(K) .
$$

\section{A geometric characterization}

We will use Proposition 4.2 to characterize uniformly quasiconformally homogeneous hyperbolic manifolds in dimensions three and above.

Theorem 1.3. Suppose that $n \geq 3$. A hyperbolic $n$-manifold is uniformly quasiconformally homogeneous if and only if it is a regular cover of a closed hyperbolic orbifold.

Proof of Theorem 1.3. Proposition 2.7 gives that every regular cover of a closed hyperbolic orbifold is uniformly quasiconformally homogeneous.

Suppose that $M=\mathbb{H}^{n} / \Gamma$ is a $K$-quasiconformally homogeneous hyperbolic $n$-manifold with $n \geq 3$. Let $G=\operatorname{Isom}_{+}(M)$. Theorem 1.1 gives that $\Lambda(\Gamma)=\mathbb{H}^{n}$, so $\Gamma$ is non-elementary. Lemma 2.3 then implies that $G$ acts properly discontinuously on $M$. Fix $x_{0} \in M$. If $y \in M$, then there exists a $K$-quasiconformal map $f: M \rightarrow M$ such that $f\left(x_{0}\right)=y$. Proposition 4.2 implies that there exists $g \in \operatorname{Isom}_{+}(M)$ such that $d\left(g\left(x_{0}\right), y\right) \leq \psi_{n}(K)$. It follows that

$$
\operatorname{diam}(M / G) \leq \psi_{n}(K),
$$

so $Q=M / G$ is a closed hyperbolic orbifold and $M$ is a regular cover of $Q$.

The following lemma provides a plethora of counterexamples to Theorem 1.3 in dimension 2 . Notice that any hyperbolic surface which is quasiconformally homeomorphic to a regular cover of a closed hyperbolic orbifold is also uniformly quasiconformally homogeneous. The basic idea is that any noncompact regular cover of a closed hyperbolic 2-orbifold has an infinite-dimensional quasiconformal deformation space and that not all of these surfaces can be regular covers of closed orbifolds.

Lemma 5.1. Let $F$ be a noncompact surface, other than $\mathbb{H}^{2}$, which is a regular cover of a closed hyperbolic 2-orbifold $Q$. Then there exists a hyperbolic surface $F^{\prime}$ which is quasiconformally homeomorphic to $F$, which is not isometric to a regular cover of a closed hyperbolic orbifold. 
Proof of Lemma 5.1. We first notice that since $F$ is noncompact and covers a closed hyperbolic orbifold, its isometry group $G=\operatorname{Isom}(F)$ is infinite. Let $\alpha_{1}$ be a simple closed geodesic on $F$, and let $L$ denote its length. Let $A_{1}$ be an annular domain in $F$ with $\alpha_{1}$ as its central curve. We may choose $A_{1}$ so that it is conformal to an Euclidean annulus of modulus $M$ the central circle of which is identified with $\alpha_{1}$. Since $G$ is infinite and acts properly discontinuously on $F$, there exists an infinite collection $\left\{g_{1}=\mathrm{id}, g_{2}, g_{3}, \ldots, g_{m}, \ldots\right\}$ of elements of $G$ such that $A_{j}=g_{j}\left(A_{1}\right)$ is disjoint from $A_{k}$ for all $j \neq k$. Let $\alpha_{j}=g_{j}\left(\alpha_{1}\right)$. We form $F^{\prime}$ by cutting along $\alpha_{j}$ for each $j$ and inserting an Euclidean annulus of modulus $M / j$. One may clearly construct a 2-quasiconformal map $f: F \rightarrow F^{\prime}$ which is conformal off of $\bigcup A_{j}$ and is $\left(1+\frac{1}{j}\right)$-quasiconformal on $A_{j}$.

Corollary 3.2 in McMullen [15] guarantees that the geodesic representative of each $f\left(\alpha_{j}\right)$ has length less than $L$. Let $\tilde{\alpha}_{j}$ be a lift of $\alpha_{j}$ to $\mathbb{H}^{2}$ and let $\tilde{f}: \mathbb{H}^{2} \rightarrow \mathbb{H}^{2}$ be a lift of $f$. Let $\gamma_{j}: \mathbb{H}^{2} \rightarrow \mathbb{H}^{2}$ be an isometry which moves a point on $\tilde{\alpha}_{j}$ to a fixed basepoint $x_{0}$ of $\mathbb{H}^{2}$ and let $\beta_{j}: \mathbb{H}^{2} \rightarrow \mathbb{H}^{2}$ be an isometry which moves $\tilde{f}\left(\gamma_{j}^{-1}\left(x_{0}\right)\right)$ to $x_{0}$. Consider $h_{j}=\beta_{j} \circ \tilde{f} \circ \gamma_{j}^{-1}$. Given any $\varepsilon>0$ and $T>0$, for all large enough $j$, the map $h_{j}$ is 2-quasiconformal on $\mathbb{H}^{2}$ and $(1+\varepsilon)$-quasiconformal on a neighborhood of radius $T$ of $x_{0}$. It follows that $\left\{h_{j}\right\}$ converges to an isometry, so the length of the geodesic representative of $f\left(\alpha_{j}\right)$ converges to $L$. It follows that the geodesic length spectrum of $F^{\prime}$ has an accumulation point at $L$.

The length spectrum of a regular cover of a closed hyperbolic orbifold is discrete, so $F^{\prime}$ must not be a regular cover of a closed orbifold.

\section{Uniform bounds on quasiconformal homogeneity}

In this section we show, again for dimensions $n \geq 3$, that there exists a uniform lower bound on the quasiconformal homogeneity constant. It is not known whether such a uniform bound exists in dimension $n=2$.

Theorem 1.4. If $n \geq 3$, there is a constant $K_{n}>1$ such that if $M$ is a $K$-quasiconformally homogeneous hyperbolic n-manifold which is not $\mathbb{H}^{n}$, then $K \geq K_{n}$.

We will first need a uniform lower bound on the diameter of a hyperbolic $n$-orbifold.

Lemma 6.1. For each $n \geq 2$ there exists $r_{n}>0$ such that if $Q$ is a hyperbolic $n$-orbifold, then $\operatorname{diam}(Q) \geq r_{n}$.

Proof of Lemma 6.1. It is a consequence of the Margulis Lemma, see Corollary 4.1.17 in [18], that, given $n$, there exists $\varepsilon_{n}>0$ such that if $x_{0} \in \mathbb{H}^{n}$, then any discrete subgroup of $\operatorname{Isom}_{+}\left(\mathbb{H}^{n}\right)$ generated by elements which move $x_{0}$ at most $\varepsilon_{n}$ is elementary. It follows that if $Q=\mathbb{H}^{n} / \Gamma$ and $\Gamma$ is nonelementary, then the diameter of $Q$ is at least $\varepsilon_{n} / 2$. On the other hand, if $\Gamma$ is elementary then $Q$ has infinite diameter. 
Proof of Theorem 1.4. Suppose that $M$ is a $K$-quasiconformally homogeneous hyperbolic $n$-manifold. Let $G=\operatorname{Isom}_{+}(M)$ and let $Q=M / G$. By Lemma 6.1 there exist $x_{1}, x_{2} \in Q$ such that $d\left(x_{1}, x_{2}\right) \geq r_{n}$. Choose $K_{n}>1$ so that $\psi_{n}\left(K_{n}\right)<r_{n}$. Choose lifts $y_{1}$ and $y_{2}$ of $x_{1}$ and $x_{2}$ to $M$. There exists a $K$-quasiconformal homeomorphism $f: M \rightarrow M$ such that $f\left(y_{1}\right)=y_{2}$. Proposition 4.2 implies that there exists $g \in G$ such that $d\left(f\left(y_{1}\right), g\left(y_{1}\right)\right) \leq \psi_{n}(K)$. This implies that $d\left(x_{1}, x_{2}\right) \leq \psi_{n}(K)$. Therefore, $\psi_{n}(K) \geq r_{n}$. Since $\psi_{n}$ is increasing, this implies that $K>K_{n}$.

We remark that one might attempt to prove Theorem 1.4 by bounding $d(M) / l(M)$ uniformly away from 1 for all hyperbolic $n$-manifolds (for fixed $n$.) One could then apply Lemmas 3.4 and 3.5, much as in the proof of Theorem 1.1, to establish Theorem 1.4. The next lemma demonstrates that this approach fails. In particular, we produce many sequences $\left\{M_{j}\right\}$ of uniformly quasiconformally homogeneous hyperbolic manifolds where $\left\{\frac{d\left(M_{j}\right)}{\ell\left(M_{j}\right)}\right\}$ converges to 1 .

Lemma 6.2. If $M=\mathbb{H}^{n} / \Gamma$ is a closed hyperbolic manifold, then there exists a sequence $\left\{M_{j}\right\}$ of finite degree, regular covers of $M$ such that $\left\{\frac{d\left(M_{j}\right)}{\ell\left(M_{j}\right)}\right\}$ converges to 1 .

Proof of Lemma 6.2. For each $L>0$ there are only finitely many conjugacy classes of hyperbolic elements of $\Gamma$ with translation length at most $L$. Let $\left\{a_{1}, \ldots, a_{n}\right\}$ be representatives of these conjugacy classes. As $\Gamma$ is residually finite (see Malcev [11]) there exists a finite index subgroup $\Gamma_{0}$ so that $a_{i} \notin \Gamma_{0}$ for $i \in\{1, \ldots, n\}$. Let $\Gamma_{L}$ be the finite index, normal subgroup of $\Gamma$ formed by intersecting all conjugates of $\Gamma_{0}$ in $\Gamma$. In particular, $\Gamma_{L}$ does not contain any element with translation length less than $L$. If $M_{L}=\mathbb{H}^{n} / \Gamma_{L}$, then $d\left(M_{L}\right)>L$. Let $x$ be a point in $M_{L}$ such that $\operatorname{inj}_{M_{L}}(x)=d\left(M_{L}\right) / 2$ and let $D$ be the diameter of $M$. If $y \in M_{L}$, then since $M_{L}$ is a regular cover of $M$, there exists an isometry $\gamma$ of $M_{L}$ such that $d(y, \gamma(x)) \leq D$. Since

$$
\operatorname{inj}_{M_{L}}(\gamma(x))=\frac{d\left(M_{L}\right)}{2}
$$

we see that

$$
\operatorname{inj}_{M_{L}}(y) \geq \frac{d\left(M_{L}\right)}{2}-D
$$

Therefore,

$$
d\left(M_{L}\right) \geq \ell\left(M_{L}\right) \geq d\left(M_{L}\right)-2 D .
$$

If we take $L$ arbitrarily large, we obtain finite degree, regular covers $M_{L}$ of $M$ with $\frac{d\left(M_{L}\right)}{\ell\left(M_{L}\right)}$ arbitrarily close to 1 . The result follows. 


\section{A topological characterization in dimension 3}

The following theorem offers a characterization of noncompact uniformly quasiconformally homogeneous hyperbolic 3-manifolds with finitely generated fundamental group.

Theorem 7.1. Let $M$ be a noncompact uniformly quasiconformally homogeneous hyperbolic 3-manifold with finitely generated fundamental group. Then there exists a closed hyperbolic 3-manifold $N$ which fibers over the circle such that $M$ is the cover associated to the fiber.

Proof of Theorem 7.1. Theorem 1.3 implies that there exists a closed hyperbolic orbifold $Q$ such that $M$ is a regular cover of $Q$. Selberg's Lemma [17] implies that there is a finite manifold cover $Q^{\prime}$ of $Q$. Then, $M$ has a finite cover $M^{\prime}$ which is a regular cover of $Q^{\prime}$. Since $M^{\prime}$ also has finitely generated fundamental group, Theorem 3 of Hempel-Jaco [8] implies that there is a closed 3-manifold $Q^{\prime \prime}$ which fibers over the circle, such that $M^{\prime}$ is the cover associated to the fiber subgroup. In particular, $\pi_{1}\left(M^{\prime}\right)$ is a closed surface group.

Let $C$ be a compact core for $M$, i.e. a compact submanifold of $M$ such that the inclusion of $C$ into $M$ is a homotopy equivalence. (The existence of a compact core is guaranteed by Scott [16].) Since $\pi_{1}\left(M^{\prime}\right)$ has finite index in $\pi_{1}(M)$, Theorem 10.6 in [7] implies that $\pi_{1}(M)$ is a closed surface group (either orientable or nonorientable) and that $C$ is an $I$-bundle. Bonahon's theorem [3] then implies that $M$ is homeomorphic to the interior of $C$ and $M-C$ is homeomorphic to $\partial C \times(0, \infty)$. We also know that the isometry group $G=\operatorname{Isom}(M)$ is infinite.

We first suppose that $C$ is a twisted $I$-bundle. In this case $\partial C$ is connected and there cannot be a compact core for $M$ contained in $M-C$. Since $G$ is infinite and acts properly discontinuously on $M$, there exists $g \in G$ such that $g(\partial C)$ does not intersect $C$. Then $g(\partial C)$ bounds a compact core $D=g(C)$ for $M$. Since $\partial C \cap \partial D=\emptyset$ and $D$ cannot be contained in $M-C$, the core $C$ is a compact submanifold of $D$ whose boundary is contained in the interior of $D$. However, since $g$ is an isometry, $C$ and $D$ must have the same volume, which is impossible. It follows that $C$ is not a twisted $I$-bundle.

Now suppose that $C$ is an untwisted $I$-bundle, i.e. homeomorphic to $F \times$ $[-1,1]$ for some closed surface $F$. Then $M$ is homeomorphic to $F \times \mathbb{R}$. Let $S$ be the surface in $M$ which is identified with $F \times\{0\}$. If $T$ is any closed incompressible surface in $M$, then $T$ is isotopic to $F \times\{0\}$ (see Corollary 3.2 in Waldhausen [21]). In particular, every closed incompressible surface separates $M$ into two unbounded components $(M-T)_{+}$and $(M-T)_{-}$, so that $(M-T)_{+}$contains $F \times[r, \infty)$ for some $r$ and $(M-T)_{-}$contains $F \times(-\infty, s]$ for some $s$. We say that a subset $X$ of $M-T$ lies above $T$ if it is contained in $(M-T)_{+}$. Similarly, we say that a normal vector $\vec{v}$ to $T$ is upward pointing if it points into $(M-T)_{+}$.

Let $G_{0}$ be the finite index subgroup of $G$ consisting of those $g \in G$ which are orientation-preserving and take upward-pointing normal vectors to $S$ to upward- 
pointing normal vectors to $g(S)$. Since $G_{0}$ is infinite and acts properly discontinuously, there exists $g \in G_{0}$ such that $g(S)$ does not intersect $S$ and lies above $S$. The surfaces $S$ and $g(S)$ bound a submanifold $D$ homeomorphic to $S \times[0,1]$ (see Corollary 5.5 in Waldhausen [21]). Let int $(D)$ denote the interior of $D$. If $n>0$, then $g^{n}(\operatorname{int}(D))$ lies above $g(S)$, so it is disjoint from $D$, while if $n<0$, then $g^{n}(\operatorname{int}(D))$ lies below $S$, so is disjoint from $D$. Thus, $D$ is a fundamental domain for the action of the cyclic group $\langle g\rangle$ generated by $g$ on $M$. It follows that $N=M /\langle g\rangle$ is a closed 3-manifold which fibers over the circle and $M$ is the cover of $N$ associated to the fiber $p(S)$ where $p: M \rightarrow N$ is the covering map.

\section{References}

1. Apanasov, B.: Geometrically finite hyperbolic structures on manifolds. Ann. Global Anal. Geom. 1, 1-22 (1983)

2. Beardon, A., Maskit, B.: Limit points of Kleinian groups and finite sided fundamental polyhedra. Acta Math. 132, 1-12 (1974)

3. Bonahon, F.: Bouts des variétiés hyperboliques de dimension 3. Ann. Math. 124, 71-158 (1986)

4. Bonfert-Taylor, P., Taylor, E.C.: Hausdorff dimension and limit sets of quasiconformal groups. Michigan Math. J. 49, 243-257 (2001)

5. Gehring, F.W., Martin, G.J.: Inequalities for Möbius transformations and discrete groups. J. Reine Angew. Math. 418, 31-76 (1991)

6. Gehring, F.W., Palka, B.: Quasiconformally homogeneous domains. J. Analyse Math. 30, 172-199 (1976)

7. Hempel, J.: 3-manifolds. Princeton University Press, 1976

8. Hempel, J., Jaco, W.: Fundamental groups of 3-manifolds which are extensions. Ann. Math. 95, 86-98 (1972)

9. MacManus, P., Näkki, R., Palka, B.: Quasiconformally homogeneous compacta in the complex plane. Michigan Math. J. 45, 227-241 (1998)

10. MacManus, P., Näkki, R., Palka, B.: Quasiconformally bi-homogeneous compacta in the complex plane. Proc. London Math. Soc. 78, 215-240 (1999)

11. Malcev, A.: On faithful representations of infinite groups of matrices. Mat. Sb. 8(1940), 405-422, Am. Math. Soc. Translations 45, 1-18 (1965)

12. Martin, G.J.: On discrete Möbius groups in all dimensions: A generalization of Jørgensen's inequality. Acta Math. 163, 253-289 (1989)

13. Maskit, B.: Kleinian Groups. Springer-Verlag, New York, 1987

14. McMullen, C.T.: Renormalization and 3-Manifolds which Fiber over the Circle. Princeton University Press, 1996

15. McMullen, C.T.: Complex earthquakes and Teichmüller theory. J. Am. Math. Soc. 11, 283-320 (1998)

16. Scott, P.: Compact submanifolds of 3-manifolds. J. London Math. Soc. 7, 246-250 (1974)

17. Selberg, A.: On discontinuous groups in higher dimensional symmetric spaces. In: Colloquium Function Theory, Tata Institute, 1960

18. Thurston, W.P.: Three-dimensional Geometry and Topology. Princeton University Press, 1997

19. Väisälä, J.: Lectures on $n$-dimensional Quasiconformal Mappings. Lecture Notes in Mathematics 229, Springer-Verlag, New York, 1971

20. Vuorinen, M.: Conformal Geometry and Quasiregular Mappings. Lecture Notes in Mathematics, 1319. Springer-Verlag, Berlin, 1988 
21. Waldhausen, F.: On irreducible 3-manifolds which are sufficiently large. Ann. Math. 87, 56-88 (1968)

22. Wielenberg, N.: Discrete Moebius groups: fundamental polyhedra and convergence. Am. J. Math. 99, 861-877 (1977)

23. Yamada, A.: On Marden's universal constant of Fuchsian groups. Kodai Math. J. 4, 266-277 (1981) 